\title{
EDUCACIÓN COMO PRÁCTICA SOCIAL: \\ LA CUESTIÓN DEL OTRO Y SU RECONOCIMIENTO
}

\section{Education as social practice: \\ the question of the other and their recognition}

\author{
DARWIN JOAQUI ROBLES \\ Universidad de San Buenaventura/Bogotá-Colombia \\ daryjoaqui@hotmail.com \\ Código Orcid: http://orcid.org/0000-0002-4833-7652
}

\author{
DORYS NOEMY ORTIZ GRANJA* \\ Pontificia Universidad Católica del Ecuador/Quito-Ecuador \\ dortiz107@puce.edu.ec \\ Código Orcid: http://orcid.org/000-0003-0617-0361
}

\begin{abstract}
Resumen
El presente artículo aborda la cuestión de la educación como práctica social en la que es importante considerar la cuestión del otro y de su reconocimiento. Los dos temas son importantes debido al aumento de problemas de acoso escolar en las instituciones educativas, que señalan una falla en el reconocimiento al otro debido, en gran parte, al impacto del uso de la realidad virtual, con la que las ideas se transmiten como mensajes dirigidos a una comunidad sin rostro y que permanece anónima en las redes sociales. La reflexión se realiza de manera analítica; se apoya en las ideas de Lévinas y Ricoeur y se enriquece con los aportes de otros autores diversos. El texto se organiza alrededor de dos ejes esenciales: el otro y el reconocimiento, para lo cual, inicia con un análisis de la cuestión de la alteridad, mediante la oposición yo-otro, que se resuelve en una síntesis en la que el yo integra aspectos del otro, proceso que se realiza gracias a la percepción y la conciencia y que se funda en la relación primaria de la madre con su hijo. Luego se aborda el tema del reconocimiento a partir de tres fuentes: el amor, el jurídico y la estima social; durante todo el trabajo se realicen reflexiones y analogías con respecto a la educación. Finalmente, se introducen varias conclusiones respecto a la educación, tanto en Colombia como en Ecuador que está más orientada hacia los aspectos formales en lugar de hacia los aspectos más humanos.
\end{abstract}

\section{Palabras claves}

Otro, reconocimiento, Lévinas, Ricoeur.

Forma sugerida de citar: Joaqui Robles, Darwin Ortiz \& Granja, Dorys Noemy, (2017). Educación como práctica social: la cuestión del otro y su reconocimiento. Sophia: colección de Filosofía de la Educación, 23(2), pp. 195-218

* Licenciado en Filosofía, cursa estudios de Maestría en Filosofía Latinoamericana en la Universidad San Buenaventura, Bogotá- Colombia; docente en el Colegio El Carmelo de Bogotá.

** Psicóloga Clínica, terapeuta familiar sistémica, docente de la Pontificia Universidad Católica del Ecuador, Quito-Ecuador. 


\begin{abstract}
This article addresses the issue of education as a social practice in which it is important to consider the question of the other and their recognition. The two themes are important due to the increasing problems of bullying in schools, indicating a failure in the recognition of the other due, in large part, to the impact of the use of virtual reality, where ideas are transmitted as messages directed to a community without a face and which remains anonymous in social networks. The reflection is done in an analytical way. It is based on the ideas of Levinas and Ricoeur and is enriched by the contributions of other various authors. The text is organized around two key axes: the other and recognition, which begins with an analysis of the question of otherness, by the opposition I - other, which is resolved in a synthesis in which the I integrates aspects of other, processing that is carried out thanks to the perception and consciousness, and that is fund in the primary relationship of mother with her son. Then addresses the issue of recognition from three sources: the love, the legal and social esteem; during all the work made reflections and analogies with regard to education. Finally, several conclusions regarding education, both in Colombia and in Ecuador which is more oriented towards the formal aspects rather than the more human aspects are introduced.
\end{abstract}

Keywords

Other, recognition, Lévinas, Ricoeur.

\title{
Introducción
}

Este artículo aborda el tema de la educación como práctica social pero trata la cuestión del otro y su reconocimiento con más detalle, considerándolos como elementos centrales de una práctica educativa relevante. Para llevar a cabo la tarea, se usan los aportes de Lévinas y Ricoeur esencialmente, los cuales se ven enriquecidos y ampliados con las ideas y propuestas de otros autores.

El objetivo es re-pensar la cuestión del otro en la educación y la importancia de su reconocimiento como tal para favorecer el proceso de aprendizaje-enseñanza. Esta cuestión es muy importante puesto que en los últimos años ha aumentado el nivel de situaciones de acoso escolar que generan un conflicto a nivel individual, familiar y social y tienen profundas repercusiones en el desarrollo emocional y social de los estudiantes. Una persona sometida a una situación de acoso enfrenta una falta total de reconocimiento hacia su calidad de individuo con sus límites y sus valiosos recursos; como consecuencia de ello, puede presentar afectaciones psicológicas como depresión, aislamiento e incluso baja en su rendimiento académico. Además, se sabe que el acosador tiene un perfil, cuya característica principal es la falta de empatía y de reconocimiento hacia los demás, por eso los hace víctimas de su poder.

En la medida en que este problema es cada vez más frecuente en el ámbito escolar, se establece la importancia de reflexionar sobre el tema del reconocimiento del otro, cuyo rostro se encuentra con frecuencia en 
las aulas de clase, en las que es importante la capacidad de darse cuenta de las condiciones de cada estudiante y aceptarlas tal cual se manifiestan.

Debido a estas consideraciones, este tema es de actualidad puesto que los niveles de alteridad van disminuyendo conforme cada persona se abstrae cada vez más del contexto en el que vive y se limita a tener interacciones a través de medios electrónicos; lo cual conduce a vivir realidades que son virtuales pero que no los ponen frente al otro en sus condiciones y limitaciones.

Con esta reflexión se pretende argumentar y sostener la idea de que se requiere el desarrollo de la habilidad para reconocer al otro, lo cual pasa por la clara conciencia sobre la identidad propia y la percepción del otro en su calidad de tal.

La reflexión se realiza de forma analítica ampliando las ideas y argumentos a partir de dos ejes esenciales: el otro, su reconocimiento y las implicaciones para la educación, líneas en las cuales se organiza el texto. El artículo termina con ciertas conclusiones respecto a los temas tratados.

\section{Del Yo al Otro: el concepto de alteridad y el principio de individuación}

Un individuo participa materialmente de una misma especie que incluye a los demás seres humanos pero, formalmente, es único, indivisible e irrepetible; con lo que se puede decir que existe per se la posibilidad de hablar de un yo pero también de un otro diferente formalmente a uno mismo.

Partiendo de esta idea, es pertinente y apropiado relacionar estos elementos con el término de Alteridad propuesto por Lévinas, entendida como la presencia del otro en el yo: "La alteridad es la idea de lo Infinito en el Mismo, en el Yo [...] la alteridad se cifra en la relación entre lo Infinito y el Yo; esta relación es metafísica, porque está más allá de todo más allá conceptual” (Quesada, 2011, p. 1).

La comprensión de la alteridad; es decir de la relación yo-otro, se relaciona con el principio de individuación, puesto que cada "yo" deviene un "sí-mismo" gracias a esta relación. Según Pérez-Estévez este principio es entendido como "la imposibilidad de división en un individuo; no se puede considerar formalmente la repetición de éste pues dejaría de ser uno y por ende individual (Individuum materiale non potest dividi in plura eiusdem naturae)" (Pérez-Estévez, 1996, pp. 783-784). En consecuencia, también se puede considerar que es aquí donde lo que prima no es la igualdad o semejanza sino la diferencia. 
Así, el principio de individuación puede significar, partiendo de Escoto, cierta independencia personal, entendida como aquel estado que el individuo puede obtener per se, en su estado existencial e itinerante; se trata de una existencia única, irrepetible, absolutamente insubstituible de la cual se deriva la dignidad del individuo; no puede ser interpretada como autismo sino como condición de posibilidad de cualquier relación auténtica de igualdad ya que: "Ser individuo implica ser, en cuanto individuo, radicalmente distinto, diferente y formalmente otro respecto a los demás individuos de la misma especie, aunque simultáneamente implique ser idénticos, en cuanto pertenecientes a la misma especie" (PérezEstévez, 1996, p. 784).

El Otro acontece per se, se muestra, es visible pero no es un Yo que se mide por medio de la aproximación, sino que es absolutamente diferente y su acontecer mismo no depende de uno; es decir, el Otro no aparece porque un Yo lo decida o determine; aparece como individual e indivisible, como "presencia en nosotros. Presencia que significa necesariamente existir, y un existir separadamente" (Quesada, 2011, p. 394). Ese Otro es lo que responde a aquello que uno no es, a aquello que es anterior a uno mismo y gracias a lo cual, una persona es quien es; es decir, el otro le constituye de tal forma que, si se niega su existencia se niega la existencia de uno mismo.

A partir de estas reflexiones, también se pueden considerar las diferencias entre el principio de individuación de Escoto y el concepto de alteridad en Lévinas, pues para Escoto el Principio de Individuación se explica desde un origen ontológico, es decir, preexistente a toda concepción general con tendencia al universalismo. Lo que importa es el Yo en primera instancia. Se podría hablar de otros "Yoes" pero no como prioridad: "Duns Escoto va a plantearse el origen ontológico de la individualidad" (Pérez-Estévez, 1996, p. 783).

Dicho principio se sitúa en determinar el por qué la importancia de aclarar la indivisibilidad del Yo, mientras que para Lévinas la importancia es ética antes que ontológica. La Alteridad (en mutua pertenencia con el Yo) no es de carácter netamente ontológico sino que tiene un instintivo ético. "la relación entre lo Infinito (Otro) y el Mismo (Yo) es una relación ética...” (Quesada, 2011, p. 395). Si bien es claro que en los dos autores están en ambos campos (tanto ontológico como ético) discrepan en la primacía de los mismos al momento de hablar del Yo como del Otro, implícita o explícitamente.

Considerando entonces estos principios de alteridad y de individuación, es posible pensar algunas implicaciones para la educación. 


\section{La cuestión del otro y sus implicaciones en la educación}

El otro es un hecho concreto, tiene una existencia específica y particular; no es un ente imaginario o abstracto, producto de alguna disquisición febril, de un filósofo sonámbulo. Lévinas reconoce que "la ontología cartesiana [...] concibe el Mismo como totalidad que integra a cualquier Otro y esto suprime la trascendencia" (Lévinas, 2008, p. 170). Entonces, se trata que el sí mismo y el otro constituyen una unidad indivisible, ¿cómo es esto posible?

No se trata de una unidad física puesto que cada persona tiene ciertas particularidades biológicas que le son propias; si se piensa incluso genéticamente, cada ser humano es único, tal vez comparta cierto nivel de rasgos heredados con otros seres humanos, incluso con otras especies, pero aun así, no hay dos seres iguales en el mundo. Más bien, se trata de un proceso de integración, como se explica a continuación.

El ser humano "está en el mundo y es en el mundo que se conoce" (Merleau-Ponty, 1994, p. 11) ya que "la realidad es un tejido sólido" (Merleau-Ponty, 1994, p. 10), por lo que la percepción, tanto de sí mismo, como del otro y del mundo alrededor no se limita a una síntesis en el nivel de los juicios o de los actos; está llena de cambios y variaciones que se sitúan en el mundo y se distinguen de los ensueños y de la imaginación, gracias a la actividad de la conciencia, necesaria para percibir al otro y, posteriormente, reconocerlo.

Este proceso comienza con la percepción, proceso psicológico por el que cada persona recibe la impresiones del mundo, de los demás seres y cosas y de sí mismo y las organiza en su mente (Gross, 2012), para lo cual es necesaria la conciencia. Siguiendo a Lévinas, entonces, se puede afirmar que "tener conciencia es estar en relación con lo que es" (Lévinas, 1977, p. 183); es tener la capacidad de asumir(se) en cuanto ser en el mundo, mantener una relación con el otro y reconocerlo como tal.

El pensador y filósofo Merleau-Ponty (1994) parte de la idea de que "Toda consciencia es consciencia de algo" (p. 17), lo cual ya fue evidenciado por Kant cuando afirmó: "la percepción interior es imposible sin percepción exterior; que el mundo, como conexión de fenómenos, se anticipa a la consciencia de mi unidad, es para mí el medio de realizarme como consciencia" (Merleau-Ponty, 1994, p. 17).

Esta conciencia accede al otro a través de la percepción que se tiene de él, lo considera en su facticidad; sin embargo, hay que considerar que existen diferencias entre los otros, según se trate de objetos o personas quiénes, a su vez, también tienen una conciencia. Así, se establece una 
relación que implica una mutua conciencia en dos niveles: de sí mismo como ser individual y, del otro como diferente a uno mismo.

Entonces, entre el otro y uno mismo se construye una relación que se puede mantener sin que haya nexo entre ambos (Lévinas, 2008); sin embargo, esto no sucede en la educación, como se verá más adelante, puesto que, en el proceso educativo, docentes y estudiantes entablan necesariamente una relación con las personas que los rodean, a partir de la cual, logran una percepción de sus condiciones, tanto a nivel personal como a nivel de los demás.

De esta manera, es posible percibir al otro e intentar comprenderlo en su forma particular de existir, con sus características propias e individuales. El proceso de captar estas características, ha recibido el nombre de reducción fenomenológica que contribuye a alcanzar una conciencia 200 trascendental "ante la cual el mundo se desplegaría en una transparencia absoluta, movido de cabo a cabo por una serie de apercepciones que el filósofo tendría por misión reconstituir a partir del resultado de las mismas" (Merleau-Ponty, 1994, p. 11).

Por ende, para entender la dinámica entre el yo y el otro se requiere la existencia de una visión en tres niveles (Merleau-Ponty, 1994): de sí mismo, del otro y de los demás sobre uno mismo. Estos tres niveles de experiencia solo son posibles cuando cada persona reconoce que experimenta su propia existencia en sí mismo ante los demás. Cada persona es un otro que no puede ser reducido a una simple percepción. Su esencia es irreductible y se pertenece a sí misma, ya que solo se capta aquello que está al alcance de la propia conciencia y de aquello que el otro deja trascender, razón por la cual, "es preciso pensar en la heteronomía del Otro en el Mismo, donde el Otro no domina al Mismo sino que le despierta y le desilusiona" (Lévinas, 2008, p. 171).

Así, cada una de las personas está, parcialmente, definida por los demás individuos que las rodean, ya que ambos perciben los objetos y los individuos alrededor y, a la vez, cada uno impulsa a que el yo perciba ciertas cosas y tenga conciencia de ellas, debido a sus características particulares de forma, color, tamaño y constitución (Morris \& Maisto, 2010). Se trata así de un proceso continuo de interacción, que también se manifiesta en la educación: un docente tiene una cierta imagen sobre sus estudiantes y con ello resalta algunos rasgos y desacredita otros; similar situación ocurre con los estudiantes.

Cada uno construye así, una representación que surge de la percepción que se tiene del otro pero que es construida por uno mismo. En esta situación, el yo no está opuesto al Otro más bien lo integra como 
parte constituyente de sí mismo (Lévinas, 1977); en consecuencia, el otro no es una alteridad relativa puesto que existe y tiene sus propias características que son susceptibles de ser percibidas, sino más bien, la contraposición yo-otro se resuelve en una síntesis, en la que el otro se integra en el sí mismo de forma articulada y armoniosa (Guidano, 1999).

Vinculando esta idea con la educación, en el proceso de interacción docentes-estudiantes, unos y otros integran rasgos o niegan otros que les ayudan a constituir su identidad profesional, con lo cual, el otro es percibido como ser-en-el-mundo:

... Como generador de una dimensión de realidad irreducible y única, lo que implica el aceptar e interrogarse sobre el mundo del otro. Y es en este proceso de ponerse en el lugar del sí mismo del otro, por el cual yo sustento en mí las pretensiones del otro como llamada de una interioridad que pide ser comprendida, que yo me cuestiono a mí mismo, mi orden corriente, mi modo de vivir y sentir (Ponce, 2005, p. s.r.).

Con ello, se llega a la consideración propuesta por Lévinas respecto al otro cuya mejor expresión, de tal forma que sobrepasa al otro en mí, se denomina Rostro (Sudar, 1981) y que está representado por el pobre, la viuda, el huérfano y el extranjero, como ejemplos paradigmáticos. En todos estos casos, su reconocimiento, implica admitir su derecho a la vida. "Solo es posible el encuentro y el reconocimiento del trascendente, por y a través del rostro del otro" (Sudar, 1981, p. 95).

Según Navarro (2008, p. 181) sólo la concepción lingüística de Lévinas y, en particular, el término "expresión" aporta las claves para aproximarse a la cuestión del "rostro", ya que es gracias a la "expresión" que cada persona se presenta a sí misma; un rostro inexpresivo impide el acercamiento y en consecuencia niega el acceso al otro; un rostro expresivo, en cambio facilita el acercamiento y la interacción; esto ya se puede percibir desde la más tierna infancia en la relación entre el bebé y su madre, ya que es a través del rostro y de sus expresiones que el niño accede al mundo materno y viceversa; sin expresiones, el mundo estaría vacío de sentido y de emociones.

Dicho intercambio también acontece en el encuentro educativo en el cual, el rostro de los docentes, así como el de los estudiantes muestra mucho más de lo que expresan las palabras. El encuentro educativo no solo requiere de contenidos y temas a estudiar, también demanda una comprensión de aquello que los rostros manifiestan: sus anhelos y sueños así como también sus angustias y temores.

Todos estos aspectos se muestran en la "expresión" que, según Lévinas permite concebir el "rostro" como una primera aproximación al 
lenguaje, ya que es, en esencia, dialógico, facilita el intercambio y la comunicación entre las personas, por lo que "no es sólo que la presencia del otro garantice la posibilidad de toda comunicación, sino que también marca su inicio" (Navarro, 2008, p. 181).

El rostro es el primer contacto que una persona tiene con otra y, en consecuencia, representa un polo significativo y opuesto respecto al yo. Así, el docente entra en contacto, en primera instancia con una serie de rostros que poco a poco van surgiendo y demarcándose en su individualidad y particularidad.

Así como la presencia del rostro humano contribuye a que el bebé se dé cuenta de que hay alguien más allá de sí mismo (Papalia, Wendkos, \& Duskin, 2005), así, el yo (el bebé en el ejemplo) accede a su identidad al tomar conciencia de que es diferente a aquel rostro que percibe $y$, 202 en el mismo momento, se da cuenta de la alteridad, al percibir un rostro diferente al propio que, además, tiene un cierto grado de expresividad; intercambio que genera una nueva lógica, completamente diferente: "La relación de alteridad parte de la capacidad del "rostro", de la mera presencia del otro, de cuestionar los poderes y la autoridad de la conciencia del yo" (Navarro, 2008, p. 183).

El rostro expresa la presencia del otro y es el primer elemento gracias al cual se tiene contacto con él. Se trata de una señal Gestalt, conformada esencialmente por un triángulo: frente, ojos y nariz que constituyen la fuente más primitiva de información para el recién nacido (Spitz, 1972). El rostro, inicialmente expresivo en gestos y miradas, puede articular, además, el lenguaje verbal lo que constituye un segundo nivel de expresión que puede apoyar o refutar aquello que se transmitió inicialmente en el rostro.

Según Spitz (1972) durante los primeros seis meses de vida el niño se encuentra en un estado de recepción cenestésica, que se afirma conforme pasan los meses en una forma de percepción particular que genera respuestas profundas y globales. Se trata así de "una comunicación no verbal, no dirigida, expresiva" (Spitz, 1972, p. 41) que constituye el modelo y la base sobre la que se elaboran y construyen las futuras expresiones y reconocimientos del rostro y del otro, incluyendo las que aparecen entre docentes y estudiantes.

Es por esto, que se considera que la relación yo-otro es asimétrica y diacrónica: asimétrica puesto que los rostros expresan diferentes elementos que pueden armonizarse pero también pueden perturbarse mutuamente; y diacrónica ya que existe un intervalo de tiempo entre un rostro y el otro (Sudar, 1981, p. 99): aun cuando el rostro del bebé coincida con el 
de su madre, en el momento actual; sin embargo, el rostro de ella existió previamente.

En este proceso, el tiempo es de suma importancia ya que cuando se lo considera en la relación entre uno y el otro "surge la trascendencia a partir de la responsabilidad hacia el prójimo en la proximidad de los demás" (Lévinas, 2008, p. 169). En consecuencia, el encuentro yo/otro facilita el reconocimiento y la alteridad y, al mismo tiempo, la separación yo/otro favorece la diferenciación y la trascendencia, ya que separarse es trascender.

Gracias a la conciencia personal que cada persona tiene de sí misma, puede ponerse en contacto con el Otro; sin embargo, la construcción y consolidación de la propia identidad es previa a este encuentro (Feldman, 2007):

Nosotros tomamos nuestro destino en manos, nos convertimos en responsables de nuestra historia mediante la reflexión, pero también mediante una decisión en la que empeñamos nuestra vida; $y$ en ambos casos se trata de un acto violento que se verifica ejerciéndose (MerleauPonty, 1994, p. 20).

Sin embargo, la identidad personal se ve afectada por la relación yo/otro en la que se construye la historia, en la que, toda palabra y gesto; es decir, todas las expresiones de los rostros humanos, tienen un significado y expresan cierta toma de posición. Así, en la educación, se plantea el reto importante de asumir las diferentes perspectivas propuestas por los rostros humanos, hasta llegar al eje mismo del significado existencial o lo que Husserl ha denominado como una "génesis del sentido": "Por estar en el mundo estamos condenados al sentido; y no podemos hacer nada, no podemos decir nada que no tome un nombre en la historia" (MerleauPonty, 1994, p. 19).

Para alcanzar dicho sentido, se requiere un reconocimiento del otro, en tanto se expresa en el rostro que se percibe, así como también un reconocimiento de sí mismo y de lo que dicho rostro le dice a uno mismo.

Las implicaciones en la educación, de lo que se ha manifestado en los párrafos anteriores se esbozan a continuación.

\section{Implicaciones para la educación}

Tanto el docente y los estudiantes son una realidad concreta, tienen sus propias características positivas y también sus limitaciones y, durante el proceso educativo, entran en relación, por lo que su presencia afecta y se ve afectada por lo que los demás manifiestan o la forma en que se comportan. 
Tanto docentes como estudiantes tienen percepciones diversas de sí mismos, de los demás en la interacción y del proceso que los ha reunido. Su percepción particular está configurada por los matices y experiencias que cada uno ha tenido a lo largo de su ciclo vital y que se ponen en juego durante el encuentro diario en las horas de clase.

De igual manera, ambos: docentes y estudiantes tienen una conciencia, más o menos clara, más o menos desarrollada de las implicaciones del proceso y del encuentro para sí mismo y para los demás. Esta conciencia contribuye a que cada persona aporte una parte de sí al proceso, pero también se reserve ciertos aspectos que puede considerar personales, propios o innecesarios de manifestar en el encuentro.

Esta conciencia se revela en el mayor o menor conocimiento que cada participante tiene de sí mismo, de las personas que le rodean y del 204 proceso que los ha reunido. Todos estos elementos tendrán una influencia decisiva en la construcción de la identidad profesional -en el caso de los estudiantes- o el reforzamiento de ciertos rasgos de la identidad -en el caso del docente-. Razón por la cual, no se puede negar la influencia que estos rasgos y la conciencia que se tiene de ellos, sobre el proceso que reúne a docentes y estudiantes en las aulas de clase.

Se trata de un juego de esencias que mantienen cierto grado de conexión entre ellas, pero al mismo tiempo tienen características propias que las distinguen y constituyen la identidad particular de cada uno. Sin embargo, es importante señalar que el otro es previo a cualquier conciencia, percepción o representación que se tenga de él; existe aún antes de pensar acerca de él; sin embargo, existe en relación con uno mismo, cuando uno se aproxima a él e intenta percibirlo y reconocerlo.

Así, la educación se trata de un encuentro posible entre el propio ser y el de los demás, en el cual se revela tanto de uno como del otro, en lo que ambos manifiestan -como se ha visto, esencialmente a través del rostro y la expresión-, pero a la vez, cada uno tiene ciertas ideas acerca de los demás, de lo que piensan, sienten o plantean y que requiere un reconocimiento.

\section{La cuestión del reconocimiento en relación al otro}

En la búsqueda de la propia identidad que solo termina cuando se reconoce el valor que tiene la interacción con el otro y lo que puede aportar al propio proceso, es necesario e indispensable el reconocimiento que se puede tejer en una relación basada en la alteridad. 
Ricoeur afirma que el reconocimiento no solo se obtiene a partir de un conflicto o de una lucha, sino también a partir del diálogo y el consenso; parte de la hipótesis que: "En el reconocimiento mutuo termina el recorrido del reconocimiento de sí mismo" (Ricoeur, 2006, p. 238); para lograrlo, usa un esquema de análisis que parte de "tres modelos de reconocimiento intersubjetivo" relacionados con el amor, el derecho y la estima social, que también -siguiendo la misma lógica- se amplían a continuación.

\section{Primer modelo de reconocimiento: el amor}

Al señalar la relación yo-otro más básica y fundante, se ha hecho referencia al vínculo madre-hijo y su impacto en la constitución de la identidad propia a través de la expresión que aparece en un rostro y el reconocimiento de la alteridad al darse cuenta de que existe otro diferente a uno mismo.

Así, el primer modelo de reconocimiento se basa en este vínculo que tiene como pilar fundamental al amor y que, posteriormente, abarca todas las relaciones afectivas que constituyen al sujeto, comenzando por la familia, luego la amistad para culminar con la pareja, que conforman la base y la fuente del reconocimiento o de su ausencia (Ricoeur, 2006).

El vínculo establecido en estas relaciones precede al reconocimiento jurídico y, su inicio se ubica, tal como se ha mencionado, en la primera relación del sujeto con su madre que constituye la base y el modelo sobre la cual se estructuran las relaciones futuras de los seres humanos.

Además, hay que tener en cuenta que la relación de amor adulta se mueve entre dos polos: el de la cercanía y el de la distancia, razón por la cual, el ser humano se estructura en un continuum en permanente equilibrio entre la fusión y la independencia. El beneficio principal de este recorrido es el logro de la pertenencia en el polo de la cercanía y la recuperación de la capacidad de independencia en el polo de la distancia.

Además, tiene otro beneficio, a menudo ignorado por ser más doloroso, pero necesario si cada uno desea estructurarse como ser humano, como es el de estar solo que, a la vez, aumenta la confianza en la permanencia del vínculo invisible que se ha creado y que mantiene a los miembros del grupo unidos a pesar de la distancia y de la separación (Bowen, 1998).

Esta es la base primaria del reconocimiento mutuo puesto que la madre y el hijo se reconocen mutuamente (o no lo hacen) como sujetos dignos de confianza, suficiente como para mantener el vínculo pese a la separación. De la misma forma, los amigos se aprueban mutuamente, se reconocen como dignos de confianza que mantiene la unión aun cuando no vivan juntos ni se vean todos los días; y, de igual forma, se espera que 
exista un vínculo entre el docente y los estudiantes que facilite el reconocimiento mutuo y favorezca el proceso de aprendizaje-enseñanza.

En las primeras relaciones del niño con su familia, la confianza y el reconocimiento aparecen y se mantienen gracias a la inscripción genealógica del niño en un linaje específico, que se extiende en dos direcciones: superior que indica su filiación materna y paterna, líneas que se van abriendo conforme se avanza en las generaciones hacia atrás e inferior que señala las obligaciones y derechos que cada ser humano tiene por ser parte de su linaje.

Esta ubicación asigna una posición particular a cada persona puesto que la identifica como hijo de y, en calidad de tal, aún antes de constituirse como un sujeto, merecedor de un reconocimiento social de sus derechos; por lo tanto, forma parte de una línea específica de transmisión puesto que su nacimiento determina que se lleve a cabo una transferen206 cia: de vida, de la leyenda familiar, de la herencia, de un nombre que, a su vez, favorece el reconocimiento del lugar particular que cada uno ocupa en su linaje. Esto permite a cada individuo proyectarse más allá de sí mismo, a la búsqueda del reconocimiento en otros planos, esencialmente, el jurídico y el social (Ricoeur, 2006).

Similar esquema puede ser adaptado a la situación educativa, en la que cada estudiante se inscribe en un linaje particular -el de su profesión- y en consecuencia, se remite hacia los profesionales que lo antecedieron y que son ahora sus profesores y se dirige hacia adelante al señalar las obligaciones que surgen del ejercicio particular de su labor y, que también son transmitidas por el linaje en el que se inscribe.

De esta forma, ocurre un proceso interesante puesto que el docente reconoce al estudiante en su posición de tal y, en consecuencia, asume que, tanto uno como el otro, tienen ciertas obligaciones por tener dicho rol; pero también, el estudiante reconoce el linaje profesional del cual su profesor forma parte, tanto como docente, pero también como profesional. Así se configura y se construye la identidad profesional específica que cada estudiante asume cuando tomó la decisión -más o menos consciente y más o menos certera- de escoger tal o cual profesión.

La contraparte negativa de este reconocimiento es la humillación:

... sentida como la retirada o el rechazo de esta aprobación, alcanza a cada uno en el plano pre-jurídico de su "estar-con" otro. El individuo se siente como mirado desde arriba, por encima del hombro, incluso tenido por nada. Privado de aprobación, es como no existente (Ricoeur, 2006, p. 243).

Esta es una situación que puede convertirse en la más dolorosa en el caso de los estudiantes cuando se enfrentan a un docente que se 
relaciona de esta forma. Así, la falta de reconocimiento conduce a la sensación de inexistencia, experiencia más dolorosa cuanto más falta hace el reconocimiento.

\section{Segundo modelo de reconocimiento: el jurídico}

El primer modelo de reconocimiento alcanzado gracias al amor, se expresa socialmente en un vínculo jurídico, mediante el cual, un niño es declarado como hijo de y recibe el apellido y las historias que su linaje ha construido tanto de la familia como del niño que llega al mundo.

Aparece así, en el vínculo social establecido entre un hombre y su hijo (ya que se hereda en primer lugar el apellido del padre), un reconocimiento jurídico. Para el análisis del cual, Ricoeur se basa, esencialmente, en Hegel quien "distingue los primeros rasgos del ser-reconocido jurídicamente en el acceso a la posesión legal de los bienes materiales, en resumen, en la forma contractual del intercambio" (Ricoeur, 2006, p. 249).

Sin embargo, no se trata aquí de la asunción de bienes materiales, se trata más bien de la adquisición de elementos más intangibles que caracterizan a tal o cual linaje particular (en el caso de las profesiones) $y$, que en ocasiones, se manifiestan en símbolos como el caduceo de los médicos por ejemplo o el tridente de los psicólogos.

Cuando se trata del reconocimiento jurídico hay que considerar dos niveles: al otro y a la norma, sin la cual, sería imposible reconocer al primero; lo cual tiene, a su vez, la doble consecuencia de ampliar la esfera de los derechos reconocidos a las personas y enriquecer las capacidades que los sujetos se reconocen (Ricoeur, 2006).

De la misma forma como el vínculo amoroso genera conflictos y dificultades asociadas con la ausencia de reconocimiento, ya sea del linaje o de las características particulares de un individuo; en el modelo jurídico, la dinámica conflictual proviene de la ruptura del contrato y la búsqueda del reconocimiento por coacción legal, de tal forma, que el crimen devela la ausencia del reconocimiento y permite que la ofensa sea evaluada como un atentado contra la persona en su dimensión universal (en su calidad de ser humano). Las personas solo pueden reconocerse como sujetos de derechos si, al mismo tiempo tienen conocimiento acerca de las obligaciones normativas respecto al otro.

Cosa similar se aplica en la educación, cuando la falta de reconocimiento conduce a reivindicaciones, tanto más fuertes cuanto más dolorosa ha sido la ausencia del reconocimiento. 


\section{Tercer modelo de reconocimiento: la estima social}

El reconocimiento no se detiene solo en el nivel jurídico; alcanza un valor más elevado que adquiere tintes axiológicos cuando hace referencia a la estima: "cada una de las personas individuales mide la importancia de sus propias cualidades para la vida del otro por los mismos valores y los mismos fines" (Ricoeur, 2006, p. 256).

Por ende, la estima social está ligada estrechamente a las interpretaciones de carácter simbólico (acertadas o no) de las mediaciones sociales, siendo la educación la más importante de ellas, puesto que abarca como mínimo 16 años de la vida de un sujeto, razón por la cual, existen muchas oportunidades de ser reconocido o humillado durante todo el proceso y a múltiples niveles, a lo que se puede denominar como órdenes de reconocimiento.

Siguiendo a Ricoeur, se registran varios tipos de estos órdenes de reconocimiento (Ricoeur, 2006, p. 259), que tienen impacto sobre la declaración jurídica y que se resumen a continuación:

El complejo socioeconómico, que incluye el sistema técnico, monetario y fiscal que puede afectar el vínculo jurídico al tener falencias en su aplicación o al facilitar o dificultar que existan un reconocimiento para tal o cual segmento de la población. Este complejo puede facilitar las condiciones para que se dé el encuentro entre docentes y estudiantes (pago adecuado a los docentes, becas para los estudiantes por ejemplo) o puede dificultarlas por su carencia o mal uso.

El complejo sociopolítico, que considera al sistema jurídico, al burocrático, al democrático y a la organización paralela de la opinión pública; que de igual manera puede tener un impacto sobre el vínculo jurídico y el amoroso al establecer formas de castigo o de reconocimiento para diversos hechos llevados a cabo por las personas. Este complejo impacta de manera decisiva sobre el sistema educativo al imponer condiciones de trabajo y de organización de los diversos niveles de formación que pueden vivirse como restrictivos, al no favorecer el desarrollo de las habilidades propias de una persona. Sin embargo, también puede proponer políticas adecuadas para la re-inserción educativa en el caso de personas que tienen dificultades de diverso tipo: capacidades físicas, condición social, etc.

El complejo sociocultural, que enfrenta al sistema mediático y su impacto en la reproducción cultural de las sociedades con el sistema científico desde el punto de vista de su organización institucional. Este enfrentamiento ha determinado profundos cambios en la forma que las personas tienen de relacionarse, de tal manera que se afecta también la constitución del vínculo amoroso y determina la necesidad de construir 
formas de enfrentar nuevos problemas como los provocados por los medios de comunicación masiva: redes sociales y demás.

El ámbito educativo no es ajeno a la influencia de este complejo, especialmente, en lo que se refiere a las redes sociales que, en ocasiones, generan una ausencia de reconocimiento del otro como tal mediante mensajes ofensivos y denigrantes. Aparecen así, nuevas categorías de dificultades personales y sociales que la educación debe enfrentar de formas creativas y pertinentes.

Estos tres órdenes de reconocimiento generan un impacto diverso sobre las personas y las condiciones que les rodean y determinan, que se establezcan acreditaciones diversas según el orden implicado en la situación de una persona dada. Entonces, cuando dos personas entran en competencia y rivalidad, ellas logran que sus lugares sean acreditados o reconocidos, gracias a lo que se ha denominado como economías de la grandeza (Ricoeur, 2006, pp. 260 y ss.), para lo cual, hay que tomar en cuenta los siguientes aspectos muy importantes:

- La evaluación de las prestaciones sociales de los individuos que apelan a la idea de justicia. Para lograrlo existe una gran variedad de criterios por los cuales una persona puede ser "grande" o "pequeña" respecto a la economía de grandeza, la cual está en mayor o menor grado en coherencia con cierto tipo de éxito social.

- Si las personas en situación de protesta desean que su queja se considere "justificada", deben cumplir con los estándares o niveles establecidos en una evaluación que se realiza en base a una serie de pruebas o baterías de tests. Con esto, se plantea la necesidad de determinar las formas de justicia en base a las estrategias de justificación usadas y no sobre los valores que las personas comparten.

- Cuando se busca el bien común legítimo, el acuerdo es imposible sin una discusión previa sobre las implicaciones de los diversos temas en litigio; a esto es a lo que se denomina como grandeza cuya asignación está en estrecha relación con la evaluación realizada en base a los criterios mencionados y a las pruebas correspondientes que indican que la persona la posee.

- Finalmente, el reto más importante de esta situación es la relación entre acuerdo y desacuerdo en la asignación de la grandeza, pero también de los criterios para determinarla como tal, de tal forma que adquiera un tinte de legitimidad, sin la cual, puede ser fácilmente cuestionada. 
Estos principios pueden ser aplicados fácilmente en la educación, cuando en los últimos años han proliferado un sinnúmero de formas estandarizadas de medir todo en la educación, desde que el estudiante/ docente ingresa a la institución educativa, hasta su rendimiento y producción. Así, el reconocimiento se ha limitado a un aspecto formal como es el cumplimiento de estas normas, sin detenerse en un reconocimiento más humano y real del verdadero potencial y capacidad de los individuos.

Las formas de reconocimiento se aplican según sean los diversos órdenes de grandeza y Ricoeur (2006) señala seis, que se resumen a continuación y que el autor analiza en base al término de ciudades:

La ciudad de Dios: vinculada al principio de la gracia, permite separar la grandeza inspirada de otras formas más terrenales que pueden haber sido corrompidas por la "vana gloria", y jerarquizar los tipos de 210 bienes -como el amor-capaces de unir a los hombres.

La ciudad de la opinión: en la cual, el principio es el del reconocimiento por parte de los otros.

La ciudad doméstica: con sus principios asociados a valores como la fidelidad, la bondad, la justicia, la asistencia mutua.

La ciudad cívica: con el principio de subordinación a la voluntad general como base de legitimación de la grandeza cívica; con lo cual, las relaciones de ciudadanía se hallan mediatizadas por una relación de segundo nivel, que se basa en el contrato social que hace que todo suceda corno si cada ciudadano contratase consigo mismo y no opinase más que según él mismo.

La ciudad mercantil: cuyo principio es el vínculo mercantil que relaciona las personas por medio de bienes que todos desean.

La ciudad industrial: bajo el principio de que son los industriales quienes deben administrar las utilidades con habilidad.

Como se puede percibir fácilmente, los primeros niveles se vinculan más estrechamente con la educación, al preocuparse por los valores y la ciudadanía de los individuos en formación, así como también por su reconocimiento. Los dos últimos niveles están más relacionados con la producción y el desarrollo económico de un pueblo sin descartar su influencia decisiva en los procesos educativos.

Ricoeur (2006) señala que entre cada uno de estos mundos existen rivalidades por las pruebas que justifican las posturas de cada una de ellas; el (re)conocimiento de los otros mundos extiende los desacuerdos de las categorías hasta las pruebas mismas e incluso a su capacidad de alcanzar el bien común. 
Por ende, la confrontación y el desacuerdo pueden conducir hacia el reconocimiento pero también hacia la invalidación; esta última acarrea un litigio que afecta las pruebas pero mucho más que eso, la noción misma de la grandeza; así por ejemplo: para un industrial ¿qué valor tiene un director de orquesta? De tal forma que es una empresa azarosa volverse grande en otro mundo que no sea el propio; en especial si se desconocen las categorías usadas para valorar la grandeza.

Similar problemática enfrentan las instituciones educativas y las carreras dentro de una misma organización; así, existen disciplinas más valoradas que otras y, en consecuencia, los estudiantes también reciben mayor o menor reconocimiento según sea la carrera en la que se desempeñen.

Debido a esto, es importante la crítica para los valores que rigen cada uno de los mundos así como de los criterios que los sostienen, con lo cual, aparece la importancia de comprender en primer lugar el otro mundo para luego criticarlo; misma situación para una persona que intenta conocer a otra: hay que comprender desde qué mundo habla, cuál es su marco de referencia, para así poder (re)conocerlo.

De esta forma, es posible alcanzar un compromiso que puede abrir la puerta para el bienestar común, esto requiere que cada persona esté en la capacidad y también en el deseo de someter sus posiciones a una reflexión y cuestionamiento, considerar las posiciones del otro y emprender juntos la tarea de encontrar un punto intermedio.

Cosa rara en el mundo actual con tantas polaridades que se enfrentan y que, en ocasiones, incluso son acusadas de ser simple acomodación a una situación dada, así: "se puede considerar el compromiso como la forma que reviste el reconocimiento mutuo en las situaciones de conflicto y de disputa derivadas de la pluralidad de las economías de la grandeza" (Ricoeur, 2006, p. 266).

Sin embargo, en estas consideraciones no se toma en cuenta la cuestión de la dimensión vertical que obliga a prestar atención a la oposición entre grande y pequeño y que se opone de forma natural a la dimensión horizontal del reconocimiento en el plano de la estima de sí. Se trata del concepto de autoridad que opone a dos grupos de personas: los que mandan (que generalmente son pocos) en oposición de los que obedecen (o se supone que deben hacerlo y que son la gran mayoría).

Para comprender este concepto de autoridad, en relación con el reconocimiento, hay que recalcar que esta idea tiene un aspecto cultural innegable y que conduce, inevitablemente, al tema de la autoridad institucional: se considera que el hecho de tomar algo como verdadero implica un "valer-más" que, en calidad de "más" implica necesariamente 
que está por encima de y, en consecuencia, apunta hacia una dimensión vertical. Cosa también muy común en el aspecto institucional cuando existen organizaciones públicas que evalúan las instituciones educativas y, establecen cuáles valen más o cumplen mejor los estándares y cuáles valen menos porque tienen fallas en dicho cumplimiento. También existen relaciones docentes-estudiantes que establecen este tipo de consideraciones.

Sin embargo, existe la posibilidad de establecer una relación horizontal, en la cual, en cambio, el concepto de autoridad está vinculado con un reconocimiento tácito de superioridad de uno hacia el otro. El modelo más adecuado de este tipo de relación es el vínculo entre el maestro y el discípulo, en el que existe un reconocimiento de la grandeza del otro de forma natural: "así como una vela encendida, enciente otra, así transmite 212 el maestro, el espíritu del arte genuino, de corazón a corazón, para que se ilumine" (Herrigel, 2005, pp. 22, 23).

En la educación también se pueden percibir estos fenómenos; existen muchas posibilidades de reconocer al otro y sus recursos pero también de fallar en este reconocimiento. Desde el inicio de la vida educativa y en todo su transcurso, los docentes pueden reconocer las diferencias existentes entre sus estudiantes o intentar homogenizarlos a cualquier costo, sobretodo, cuando ellos mismos están sujetos a presiones institucionales que intentan ordenar sus acciones pero que las restringen al paso.

Por lo tanto, hay que recordar que la educación como práctica social puede ser el caldo de cultivo más aparente en el que se gesten múltiples conflictos en diversas áreas tales como las minorías negras o conjuntos culturales minoritarios como las culturas juveniles. El núcleo central de estas minorías se organiza alrededor de la necesidad del reconocimiento a una identidad personal que se refiere a su vez, a una de tipo colectivo; reconocimiento tanto más perentorio cuando se incluye una consideración temporal puesto que han sido minorías discriminadas tradicionalmente a lo largo de años, cuando no siglos (como fue el caso de los negros en su condición de esclavos) y, en consecuencia, la educación juega un papel transcendental en este proceso, al tener la puerta abierta para ofrecer un reconocimiento a su identidad y la oportunidad de ejercerla en relación con los otros.

En estos casos, la lucha por el reconocimiento personal y jurídico pone en juego consideraciones personales y remite a cada persona (tanto que no reconoce como la que no es reconocida) a confrontarse con la cuestión de la estima de sí: puesto que, por ejemplo: el reconocimiento de que las mujeres podían acceder a la educación implicaba necesariamente, 
el reconocimiento de que una mujer es igual que un hombre y, lo que es más importante aún, que éste es igual a una mujer.

En consecuencia, la identidad de las personas implicadas en esta lucha se moldea, en parte, por este reconocimiento o por su ausencia así como también por la percepción (mala o buena) que se tenga sobre el otro. La ausencia de reconocimiento alcanza la identidad de las personas y afecta, finalmente, la imagen que se hacen de sí mismos puesto que la perciben como merecedora de desprecio y condena. Esta imagen es interiorizada y se manifiesta también en forma de auto-depreciación, lo cual constituye el efecto más grave de la falta de reconocimiento (Ricoeur, 2006).

La resolución de estos conflictos opone dos políticas diferentes que inciden en las formas posibles que adoptará el reconocimiento: la de la diferencia frente a la de la igualdad universal. Ambas tienen como fundamento, la noción de dignidad, en la que también se oponen dos versiones (Ricoeur, 2006): la liberal, de igualdad, que enfatiza el estatuto racional compartido por los seres humanos y la de la diferencia que considera la afirmación de lo compartido en forma general como la expresión de una hegemonía totalizante que establece como criterios de medición y de comparación: un hombre, blanco, citadino y educado. Esta definición única es la que aparece como discriminatoria, puesto que es imposible que todos los seres humanos se identifiquen y construyan su identidad en función de ella.

Esta es la razón, finalmente por la que se plantea la idea de "saber si 'cualquier política de dignidad igualitaria, fundada en el reconocimiento de capacidad universal, tiene que ser igualmente homogeneizante"' (Ricoeur, 2006, p. 273). Esto implica que existe una negativa a reconocer que existe legitimidad en la idea de una construcción particular de proyectos individuales en el interior de un colectivo.

Esta problemática requiere la definición de una situación global y particular a la vez. El reconocimiento de los aspectos comunes compartidos por los seres humanos y al mismo tiempo, la aceptación de los diversos elementos que los hacen diferentes, en un juego en que la totalidad y la unidad son percibidas al mismo tiempo que se reconocen sus particularidades.

Esta situación revela su carácter de urgente en la medida que cada vez más, las sociedades se enfrentan a problemas de minorías que exigen su reconocimiento; lo cual implica necesariamente el reconocimiento de la sociedad como tal, en su capacidad de atender a los diversos grupos que en ella conviven y afirman sus derechos. 
De esta forma, cabe preguntarse sobre la forma en que puede surgir el reconocimiento en esta tensión global/local. Ricoeur indica que Hegel ofrece "un poderoso instrumento especulativo" (Ricoeur, 2006, p. 274) al usar los recursos de lo negativo como generadores de normatividad, de tal forma que el crimen y el desprecio se vuelven las fuentes de las cuales puede surgir el reconocimiento al otro y sus conceptos relacionados como la confianza en sí, el respeto, la estima de sí.

Este reconocimiento no puede extenderse infinitamente ya que existen límites y situaciones que requieren atención particular, por lo que también cabe preguntarse ¿Cuándo un sujeto se considera reconocido verdaderamente? (Ricoeur, 2006, p. 274), para responder a esta pregunta, cabe indicar lo que señala dicho autor:

Las experiencias de reconocimiento pacificado no pueden hacer las veces de resolución de las perplejidades suscitadas por el concepto mismo de lucha, y menos aún de resolución de los conflictos en cuestión. La certeza que acompaña a los estados de paz ofrece más bien una confirmación de que no es ilusoria la motivación moral de las luchas por el reconocimiento. Por eso, no puede tratarse más que de treguas, de claros, se diría de 'calveros', en los que el sentido de la acción sale de las brumas de la duda con el sello de la acción que conviene (Ricoeur, 2006, p. 276).

Así, la acción como tal no es suficiente para ofrecer un reconocimiento que parte de uno mismo hacia el otro. Se debe pensar en la conveniencia de dicha acción, lo cual plantea muchas más preguntas que respuestas, puesto que dicha conveniencia, a su vez, debe ser reconocida por las personas que plantean la acción a la vez, que es comprendida, aceptada $-y$, en consecuencia- reconocida por las personas que la van a experimentar en sí.

Con ello, es posible darse cuenta de que Lévinas se sitúa en el polo de comprensión de la relación yo-otro desde una fundamentación ética y anti-ontológica, en donde se ve la lucha contra la concepción del ser como asimilación; él toma distancia de la ontología tras considerar que instaura un discurso en el que el Otro es reducido a lo mismo. La apuesta del autor es buscar y dar cuenta de una cuestión crucial: "de qué manera la filosofía de la asimetría originaria entre el yo y el otro, asimetría tomada a partir de la primacía ética del otro, puede explicar la reciprocidad entre miembros desiguales" (Ricoeur, 2006, p. 204).

En la relación yo-otro, el ser resulta arrollador al punto de asimilar a sí mismo todas las diferencias posibles. Lévinas no se conforma con esta constatación y propugna un camino diferente a la primacía y dominio del 
ser, garantizada por la vía ontológica. En consecuencia, el otro no debe estar vestido con lo social, cultural, político etc.

La ética ya no está fundada en el yo sino en el otro pero teniendo en cuenta que no hay rostro del otro sin un yo que lo capte, que sería en última instancia, la exigencia humana de responsabilidad que fundamenta la propuesta ética del autor. De lo anterior, se puede decir que el otro se revela a sí mismo pero ¿cómo hace el sí mismo para conocer la revelación del otro? Desde la visión levinasiana esta afirmación posee un carácter de reciprocidad de intercambio de roles y no se debe pensar "que el mismo" es el otro, del "otro".

$\mathrm{Al}$ otro se le debe pensar como otro, pues, si se piensa como relación o reciprocidad se abriría un espacio para una ética que piensa siempre en el yo. No se debe pensar en el yo, no debe interesar, pues en Lévinas solo interesa el otro. Esto se convierte en una ética radical de la aceptación del otro. El otro es el rostro de la humanidad que pide responsabilidad.

El otro no se puede llenar de características porque en la relación ética no hay éstas, pues el otro no puede estar "vestido de...". Si esto se hace, cabría la posibilidad de plantear una metafísica de la subjetividad, es decir, una fundamentación metafísica de la "otredad" que se plantea porque el sujeto es el determinador de la presencia del otro y de la metafísica de la subjetividad; por el contrario, desde la metafísica del pensador lituano, el otro se vuelve un "subjectum" pura subjetividad. El otro es el que impone, el otro es el que funda.

En Ricoeur, en cambio, se expresa una ética de reciprocidad en términos de "Yo"-“Tu", pues hay un diálogo, hay reciprocidad es decir, el otro es un "yo" que no soy yo. Esta relación permite la posibilidad del diálogo, mientras que en Lévinas no se encuentra esa posibilidad. Lo que se encuentra en su ética es una imposición del otro hacia "yo" (mí). El otro es un yo que no soy yo. En Lévinas el otro es sagrado. En el otro hay una revelación de algo sagrado. El otro es mucho más que yo.

En Ricoeur, si el "tu" es sagrado podría decirse que no se puede sostener un diálogo con eso sagrado. Hay una imposición total del otro hacia un "yo" (mí). El "tu" generalmente posee características y rasgos determinados y en Lévinas no es posible encontrar dichas características. El otro no puede estar vestido, por eso en el ámbito de la ética no se trata de representar al otro sino más bien de "recepcionar la revelación del otro". En este sentido la presencia del otro, que es "rostro" no pasa por la representación ni pasa por el conocimiento; si pasara por el conocimiento "el rostro" del otro dejaría de ser "rostro" para convertirse en categoría. 


\section{Conclusiones}

Ambos fenómenos: conciencia y percepción, son esenciales para entrar en relación con el otro, el cual es captado en su facticidad y organicidad. El otro se revela de tal forma a la conciencia personal, en una relación de mutua influencia, puesto que se perciben solo ciertas características y no la totalidad puesto que el otro, es mucho más que aquello que se puede percibir.

La mejor forma en que el otro se expresa es el rostro y en la expresión. Al parecer, ambos son la síntesis de uno mismo y del diferente, del ajeno, de aquel ser, al que solo se accede a través de la lógica construida en uno mismo, durante el propio desarrollo, ya que solo se mira al otro, a partir de uno mismo.

El otro requiere reconocimiento en su misma constitución: su identidad; sin embargo, para lograrlo, es necesario que uno mismo reconozca el impacto que la identidad y la constitución del otro tiene sobre sí mismo.

El reconocimiento se hace desde tres instancias sumamente importantes: el amor que remite al individuo a un linaje particular; el jurídico que lo inscribe como sujeto de derechos en un entorno social específico y el de la estima social que lo convierte en sujeto de derechos en relación con otras personas que inciden sobre su propia construcción.

Este proceso no se hace sin conflictos y luchas puesto que el reconocimiento constituye la base de la construcción del sí mismo en el grupo en el cual cada persona se siente pertenecer.

Se espera que la educación pueda aportar este reconocimiento propio, al otro, en su identidad y características propias de tal forma que cada persona pueda construir su historia particular que, al fin y al cabo, se inscribe en la historia de toda la humanidad a través de los tiempos.

En los planes nacionales de Educación, tanto en Colombia como en Ecuador, se considera una gran iniciativa respecto a la manera cómo se debe educar en las aulas de clase; todo bajo un criterio objetivo "progresista" que, en muchas ocasiones, resulta excluyente pues no se consideran planos de formación y desarrollo como el contexto, los medios de educación, los docentes y, sobre todo la voz del educando. Se le denomina a la educación "integral" pero lo que se prolonga es la exclusividad y el conformismo.

Se considera a los educandos como un solo grupo, como un solo logro a alcanzar sin tener en cuenta que los contextos de vida en algunos lugares más que otros son precarios e insuficientes. Todo es pensado desde una concepción general con tendencia al universalismo. Es aún más evidente en las famosas pruebas de estado, en donde se supone que existieron garantías suficientes para lograr respuestas a dichas pruebas y a 
esto se le suma el querer pensar la educación de una misma manera para todo un contexto diverso.

Si los docentes o agentes educativos lograran acercarse por uno de los tantos caminos de la educación integral; en este caso en particular, tomando la perspectiva de Escoto como mirada educativa e individual (no individualista) e integradora, se puede dar la posibilidad de que cada educando logre ver una semejanza y mismidad entre los demás; pero de igual manera, logre identificar e integrar sus diferencias con las de los demás, capacitando así posibilidades de escuchar y ser escuchados al momento del proceso de aprendizaje-enseñanza.

Si se lograra formar personas individuales capaces de trabajar a partir de las diferencias y semejanzas ante los demás, se lograría tener, no sólo simples operarios de un sistema político en donde el interés es producir y explotar lo humano sino que esa característica individual permitiría la presencia de personas realmente humanas preocupadas por otras personas en tanto tales y no en tanto objetos de explotación.

\section{Bibliografía}

BOWEN, Murray

1998 De la familia al individuo. La diferenciación de sí mismo en el sistema familiar. Barcelona: Paidós.

FELDMAN, Robert

2007 Desarrollo psicológico a través de la vida. México: Pearson Education.

GROSS, Richard

2012 Psicología: la ciencia de la mente y la conducta. México: El Manual Moderno.

GUIDANO, Vittorio

1999 El modelo cognitivo post-racionalista. Hacia una reconceptualización teórica y crítica. Bilbao: Desclée de Brouwer.

HERRIGEL, Eugen

2005 Zen en el arte del tiro con arco. Buenos Aires: Kier.

LÉVINAS, Emmanuel

1977 Totalidad e infinito. Ensayo sobre la exterioridad. Salamanca: Sígueme.

2008 Dios, la muerte y el tiempo. Madrid: Ediciones Cátedra.

MERLEAU-PONTY, Maurice

1994 Fenomenología de la percepción. Barcelona: Planeta, De Agostini S.A.

MORRIS, Charles \& MAISTO, Albert

2010 Psicología General. Madrid: Pearson.

NAVARRO, Olivia

2008 El "rostro" del otro: una lectura de la ética de la alteridad de Emmanuel Lévinas. Contrastes. Revista Internacional de Filosofía, 177-194.

PAPALIA, Diane; WENDKOS, Sally \& DUSKIN, Ruth

2005 Desarrollo humano. México: McGraw-Hill. 
PÉREZ-ESTÉVEZ, Antonio

1996 El individuo en Duns Escoto. Táchira: Universidad de Táchira.

PONCE, Álvaro

2005 Construcción de la otredad en procesos afectivos. Una aproximación a la situación de violencia. Quito: UPS.

QUESADA TALAVERA, Balbino

2011 Aproximación al concepto de "Alteridad" en Levinas. Propedéutica de una nueva ética como filosofía primera. En: Investigaciones Fenomenológicas. Vol. 3 (Monográfico).

RICOEUR, Paul

2006 Caminos del reconocimiento. Tres estudios. México: Fondo de Cultura Económica SPITZ, René

1972 El primer año de vida. Génesis de las primeras relaciones objetales. Madrid: Aguilar.

SUDAR, Pablo

1981 El rostro del pobre. Buenos Aires: Patria Grande.

Fecha de recepción del documento: 20 de marzo de 2017 Fecha de revisión del documento: 15 de abril de 2017

Fecha de aceptación del documento: 30 de abril de 2017 Fecha de publicación del documento: 15 de julio de 2017 